\title{
SUMMING SERIES ARISING FROM INTEGRO-DIFFERENTIAL-DIFFERENCE EQUATIONS
}

\author{
P. CERONE ${ }^{1}$ and A. SOFO ${ }^{1}$
}

(Received 13 April 1995)

\begin{abstract}
By applying Laplace transform theory to solve first-order homogeneous differentialdifference equations it is conjectured that a resulting infinite sum of a series may be expressed in closed form. The technique used in obtaining a series in closed form is then applied to other examples in teletraffic theory and renewal processes.
\end{abstract}

\section{Introduction}

Differential-difference equations occur in a variety of applications including ship stabilization and automatic steering [19], the theory of electrical networks containing lossless transmission lines [7], the theory of biological systems [6] and in the study of distribution of primes [25].

The equation

$$
f^{\prime}(t)+\alpha f^{\prime}(t-a)+\beta f(t)+\gamma f(t-a)+\delta f(t+a)=0
$$

is termed a first-order linear delay, or retarded, differential-difference equation for $\alpha=0, \delta=0$ and $a>0$. For $\alpha=0, \delta=0$ and $a<0$ it is termed an advanced equation. In the case $\delta=0, a>0$ it is referred to as a neutral equation and when $\alpha=0, \beta=0, a>0$ an equation of mixed type.

Stability studies on general delay equations have been carried out in [5] and for neutral equations in [13]. Driver, Sasser and Slater [11] consider a first-order linear delay equation and for a 'small' delay they show that it exhibits certain similarities associated with an equation without delay. Numerical studies have also been carried out in which chaos has been observed [14], and Seifert [22] hints strongly at a suspected chaotic interval function associated with discontinuous delays.

\footnotetext{
'Department of Computer and Mathematical Sciences, Victoria University of Technology, PO Box 14428, Melbourne City MC, VIC 8001, Australia.

(C) Australian Mathematical Society 2000, Serial-fee code 0334-2700/00
} 
A great deal of the studies for the stability of differential-difference equations necessitate an investigation of its associated characteristic equation. Some of the early work in this area has been carried out by Pontryagin [21], Wright [28] and more recentiy by Cooke and van den Driessche [10] and Hao and Brauer [16].

The purpose of this paper is to show that, by using Laplace transform techniques together with a reliance of asymptotics, series representations for the solutions of delay equations may be expressed in closed form. The series, in its region of convergence, it is conjectured, applies for all values of the delay without necessarily relying on its association with the differential-difference equation.

Unlike some of the series that are listed as high precision fraud by Borwein and Borwein [3], the series in this paper will be shown to be exact by the use of Bürmann's theorem. The analysis also relies on the exact location of the roots of the associated transcendental characteristic equation. This technique is then applied to particular examples.

\section{Method}

Consider the first-order linear homogeneous differential-difference equation

$$
\left.\begin{array}{ll}
f^{\prime}(t)+b f(t)+c f(t-a)=0, & t \geq a, \\
f^{\prime}(t)+b f(t)=0, & f(0)=1,0 \leq t<a .
\end{array}\right\}
$$

Taking the Laplace transform and using the initial condition, results in

$$
\mathscr{L}[f(t)]=F(s)=\frac{1}{s+b+c e^{-a s}}=\sum_{n=0}^{\infty} \frac{(-1)^{n} c^{n} e^{-a n(s+b)} e^{a n b}}{(s+b)^{n+1}} .
$$

The inverse Laplace transform is

$$
\mathscr{L}^{-1}[F(s)]=f(t)=\sum_{n=0}^{\infty} \frac{(-1)^{n} c^{n} e^{-b(t-a n)}(t-a n)^{n}}{n !} H(t-a n),
$$

where the Heaviside unit function is defined to be

$$
H(x)= \begin{cases}1, & \text { for } x \geq 0 \\ 0, & \text { for } x<0\end{cases}
$$

By Laplace transform theory the solution to (1) may be written as

$$
f(t)=\frac{1}{2 \pi i} \int_{\gamma-i \infty}^{\gamma+i \infty} e^{s t}[g(s)]^{-1} d s
$$


for an appropriate choice of $\gamma$ such that all the zeros of the characteristic equation

$$
g(s)=s+b+c e^{a s}
$$

are contained to the left of the line in the Bromwich contour. Now, using the residue theorem

$$
f(t)=\sum \text { residues of }\left\{e^{s t}[g(s)]^{-1}\right\},
$$

which suggests the solution of $f(t)$ may be written in the form

$$
f(t)=\sum_{r} Q_{r} e^{s_{r} t}
$$

where the sum is over all the characteristic roots $s_{r}$ of $g(s)=0$ and $Q_{r}$ is the residue of $F(s)$ at $s=s_{r}$.

The poles of the expression (2) depend on the zeros of the characteristic equation (4), namely, the roots of $g(s)=0$. The dominant root $s_{0}$ of $g(s)=0$ has the greatest real part and therefore asymptotically $f(t) \sim Q_{0} e^{s_{0} t}$, and so from (3),

$$
f(t)=\sum_{n=0}^{\infty}(-1)^{n} c^{n} e^{-b(t-a n)} \frac{(t-a n)^{n}}{n !} H(t-a n) \sim Q_{0} e^{s_{0} t} .
$$

After some experimentation it is conjectured that:

$$
\sum_{n=0}^{\infty}(-1)^{n} c^{n} e^{-b(t-a n)} \frac{(t-a n)^{n}}{n !}=Q_{0} e^{s_{0} t}
$$

$\forall t \in R$ in the region where the series converges. Bürmann's theorem will be used, a little later, to prove the identity (6).

By the use of the ratio test it can be shown that the series in (6) converges in the region

$$
\left|a c e^{1+a b}\right|<1 \text {. }
$$

In a similar fashion, the Laplace transform from (2) may be expressed as

$$
F(s)=\frac{1}{s}\left[1+\frac{b+c e^{-a s}}{s}\right]^{-1}=\sum_{n=0}^{\infty} \sum_{r=0}^{n}(-1)^{n} b^{n-r} c^{r}\left(\begin{array}{l}
n \\
r
\end{array}\right) \frac{e^{-a r s}}{s^{n+1}}
$$

and the inverse Laplace transform may be written as

$$
f(t)=\sum_{n=0}^{\infty} \sum_{r=0}^{n}(-1)^{n} b^{n-r} c^{r}\left(\begin{array}{l}
n \\
r
\end{array}\right) \frac{(t-a r)^{n}}{n !} H(t-a r) \sim Q_{0} e^{s_{0} t}
$$


As previously, it is conjectured that

$$
\sum_{n=0}^{\infty} \sum_{r=0}^{n}(-1)^{n} b^{n-r} c^{r}\left(\begin{array}{l}
n \\
r
\end{array}\right) \frac{(t-a r)^{n}}{n !}=Q_{0} e^{s_{0} t}
$$

whenever the double series converges.

LEMMA 1. The poles of the expression (2) are all simple for the inequality (7).

PROOF. Assume on the contrary that there is a repeated root of

$$
s+b+c e^{-a s}=0 .
$$

Then by differentiation it is required that $1-a c e^{-a s}=0$, in which case $s=$ $(1 / a) \ln (a c)$. Substituting in (9) results in $\ln (a c)+a b+1=0$ and therefore $a c e^{1+a b}=1$ which violates the inequality (7). Hence all roots of (9) are simple. It is a straightforward matter to show that (9) has at most two real roots. Both Bellman and Cooke [1] and Cooke and Grossman [9] give necessary and sufficient conditions for a rearrangement of (9) to have roots with negative real part.

Now the residue $Q_{0}$ of the dominant simple root, $s_{0}=\xi$, is $1 /(1+a b+a \xi)$, where $\xi+b+c e^{a \xi}=0$ and so the expressions (6) and (8) become

$$
\begin{aligned}
\sum_{n=0}^{\infty}(-1)^{n} e^{b(t-a n)} \frac{(t-a n)^{n}}{n !} & =\sum_{n=0}^{\infty} \sum_{r=0}^{n}(-1)^{n} b^{n-r} c^{r}\left(\begin{array}{l}
n \\
r
\end{array}\right) \frac{(t-a r)^{n}}{n !} \\
& =\frac{e^{\xi t}}{1+a b+a \xi}
\end{aligned}
$$

whenever the single and double series converge in a mutual region.

LEMMA 2. (i) The single sum and the double sum in (10) are solutions to (1) in their region of convergence, for $t>a$.

(ii) The closed form expression in (10) is a solution to (1) for $t>a$.

(iii) The single and double sum in (10) are equal in their mutual region of convergence, which is no larger than that region given by (7).

Proof. Parts (i) and (ii) can be shown to be solutions of (1) by substitution. For part (iii), to show

$$
\sum_{n=0}^{\infty} \sum_{r=0}^{n}(-1)^{n} b^{n-r} c^{r}\left(\begin{array}{l}
n \\
r
\end{array}\right) \frac{(t-a r)^{n}}{n !}=\sum_{n=0}^{\infty}(-1)^{n} e^{b(t-a n)} \frac{(t-a n)^{n}}{n !}
$$


expand the left hand side to give

$$
\text { for } \begin{aligned}
& n=0: \quad b^{0} c^{0}\left(\begin{array}{l}
0 \\
0
\end{array}\right) \frac{(t-0)^{0}}{0 !} \\
& n=1:-b^{1} c^{0}\left(\begin{array}{l}
1 \\
0
\end{array}\right) \frac{(t-0)^{1}}{1 !}-b^{0} c^{1} \frac{(t-a)^{1}}{1 !} \\
& n=2: \quad b^{2} c^{0}\left(\begin{array}{l}
2 \\
0
\end{array}\right) \frac{(t-0)^{2}}{2 !}+b^{1} c^{1}\left(\begin{array}{l}
2 \\
1
\end{array}\right) \frac{(t-a)^{2}}{2 !}+b^{0} c^{2}\left(\begin{array}{l}
2 \\
2
\end{array}\right) \frac{(t-2 a)^{2}}{2 !} \\
& n=3: \quad-b^{3} c^{0}\left(\begin{array}{l}
3 \\
0
\end{array}\right) \frac{(t-0)^{3}}{3 !}-b^{2} c^{1}\left(\begin{array}{l}
3 \\
1
\end{array}\right) \frac{(t-a)^{3}}{3 !}-b^{1} c^{2}\left(\begin{array}{l}
3 \\
2
\end{array}\right) \frac{(t-2 a)^{3}}{3 !} \\
&-b^{0} c^{3}\left(\begin{array}{l}
3 \\
3
\end{array}\right) \frac{(t-3 a)^{3}}{3 !} \\
& n=4: \quad \ldots \ldots . .
\end{aligned}
$$

Summing each column results in

$$
\begin{aligned}
= & \frac{c^{0}(t-0)^{0}}{0 !}\left[\frac{1}{0 !}-\frac{(-b)(t-0)}{1 !}+\frac{(-b)^{2}(t-0)^{2}}{2 !}-\frac{(-b)^{3}(t-0)^{3}}{3 !}+\cdots\right] \\
& -\frac{c(t-a)}{1 !}\left[\frac{1}{0 !}-\frac{(-b)(t-a)}{1 !}+\frac{(-b)^{2}(t-a)^{2}}{2 !}-\frac{(-b)^{3}(t-a)^{3}}{3 !}+\cdots\right] \\
& +\frac{c^{2}(t-2 a)^{2}}{2 !}\left[\frac{1}{0 !}-\frac{(-b)(t-2 a)}{1 !}+\frac{(-b)^{2}(t-2 a)^{2}}{2 !}\right. \\
& \left.\quad-\frac{(-b)^{3}(t-2 a)^{3}}{3 !}+\cdots\right] \\
& -\frac{c^{3}(t-3 a)^{3}}{3 !}\left[\frac{1}{0 !}-\frac{(-b)(t-3 a)}{1 !}+\frac{(-b)^{2}(t-3 a)^{2}}{2 !}\right. \\
= & \left.\frac{c^{0}(t-0)^{0}}{0 !} e^{-b(t-0)}-\frac{(-b)^{3}(t-3 a)^{3}}{3 !}+\cdots\right]+\cdots \\
& -\frac{c^{3}(t-3 a)^{3}}{3 !} e^{-b(t-3 a)}+\cdots \\
= & \sum_{n=0}^{\infty}(-1)^{n} c^{n} e^{-b(t-a)}+\frac{c^{2}(t-2 a)^{2}}{2 !} e^{-b(t-2 a)}
\end{aligned}
$$

Bürmann's theorem [26] will now be used to prove the explicit form of relationship (6).

BÜRMANN's THEOREM. Let $\phi$ be a simple function in a domain $D$, zero at a point 
$\beta$ of $D$, and let

$$
\theta(z)=\frac{z-\beta}{\phi(z)}, \quad \theta(\beta)=\frac{1}{\phi^{\prime}(\beta)} .
$$

If $f(z)$ is analytic in $D$ then $\forall z \in D$

$$
f(z)=f(\beta)+\sum_{r=1}^{n} \frac{\{\phi(z)\}^{r}}{r !} \frac{d^{r-1}}{d t^{r-1}}\left[f^{\prime}(t)\{\theta(t)\}^{r}\right]_{t=\beta}+R_{n+1},
$$

where

$$
R_{n+1}=\frac{1}{2 \pi i} \int_{\Gamma} d v \int_{c}\left[\frac{\phi(v)}{\phi(t)}\right]^{n} \frac{f^{\prime}(t) \phi^{\prime}(v)}{\phi(t)-\phi(v)} d t .
$$

The $v$-integral is taken along a contour $\Gamma$ in $D$ from $\beta$ to $z$, and the t-integral along a closed contour $C$ in $D$ encircling $\Gamma$ once positively.

Application of Bürmann's Theorem The characteristic equation (4) may be shown to have a simple dominant zero at $s=0$ for $b+c=0$ and $(1+a b)>0$. Thus from (6)

$$
\sum_{n=0}^{\infty}(-1)^{n}(-b)^{n} e^{-b(t-a n)} \frac{(t-a n)^{n}}{n !}=\frac{1}{1+a b}
$$

Let $t=-a \tau, a b=-\rho$, and hence from above

$$
\sum_{n=0}^{\infty}\left(\rho e^{-\rho}\right)^{n} \frac{(\tau+n)^{n}}{n !}=\frac{e^{\rho \tau}}{1-\rho} .
$$

Equation (11) is shown to be true by applying Bürmann's theorem.

Let

$$
f(z)=\frac{e^{x z}}{(1-z)}, \quad \theta(z)=\frac{z}{\phi(z)}=e^{z}, \quad \phi(z)=z e^{-z}, \quad f(\beta)_{\beta=0}=1
$$

and it may be shown that $R_{n+1} \rightarrow 0$ as $n \rightarrow \infty$. From $f(t)=e^{t x} /(1-t)$,

$$
f^{\prime}(t)=e^{\imath x}\left(\frac{x}{1-t}+\frac{1}{(1-t)^{2}}\right)=e^{t x}\left[\sum_{j=0}^{\infty}(x+1+j) t^{j}\right]
$$

and so $f^{\prime}(t)\{\theta(t)\}^{r}=e^{t(r+x)} \psi(t)$, where $\psi(t)=\sum_{j=0}^{\infty}(x+1+j) t^{j}$. The coefficients in this expression are the same as those in a Taylor series expansion about zero

$$
\psi^{(j)}(0)=j !(x+1+j)
$$


Now let

$$
\begin{aligned}
B_{r}(t)= & \frac{d^{r-1}}{d t^{r-1}}\left[f^{\prime}(t)\{\theta(t)\}^{r}\right]=\frac{d^{r-1}}{d t^{r-1}}\left[e^{t(r+x)} \psi(t)\right] \\
= & e^{t(r+x)}\left[(r+x)^{r-1}\left(\begin{array}{c}
r-1 \\
0
\end{array}\right) \psi^{(0)}(t)+\left(\begin{array}{c}
r-1 \\
1
\end{array}\right)(r+x)^{r-2} \psi^{\prime}(t)\right. \\
& +\left(\begin{array}{c}
r-1 \\
2
\end{array}\right)(r+x)^{r-3} \psi^{\prime \prime}(t)+\cdots+\left(\begin{array}{c}
r-1 \\
r-2
\end{array}\right)(r+x) \psi^{(r-2)}(t) \\
& \left.+\left(\begin{array}{c}
r-1 \\
r-1
\end{array}\right)(r+x)^{0} \psi^{(r-1)}(t)\right] \\
B_{r}(0)= & (r+x)^{r-1}\left(\begin{array}{c}
r-1 \\
0
\end{array}\right)(x+1)+(r+x)^{r-2}\left(\begin{array}{c}
r-1 \\
1
\end{array}\right)(x+2) \\
& +(r+x)^{r-3}\left(\begin{array}{c}
r-1 \\
3
\end{array}\right)(x+3)+\cdots \\
& +\left(\begin{array}{c}
r-1 \\
r-2
\end{array}\right)(r+x)(r-2) !(x+r-1)+\left(\begin{array}{c}
r-1 \\
r-1
\end{array}\right)(r-1) !(x+r)
\end{aligned}
$$

Putting $y=x+r$ gives

$$
\begin{aligned}
B_{r}(0)= & y^{r-1}(y-r+1)+y^{r-2}(r-1)(y-r+2) \\
& +y^{r-3}(r-2)(r-1)(y-r+3)+\cdots+(r-1) ! y(y-1)+(r-1) ! y \\
= & y^{r}-(r-1) y^{r-1}+(r-1) y^{r-1}-(r-1)(r-2) y^{r-2}+(r-1)(r-2) y^{r-2} \\
& -(r-1)(r-2)(r-3) y^{r-3}+\cdots+y^{2}(r-1) !-(r-1) ! y+(r-1) ! y \\
= & y^{r}=(x+r)^{r} .
\end{aligned}
$$

Hence it follows that

$$
\frac{e^{x z}}{1-z}=1+\sum_{r=1}^{\infty} \frac{\left(z e^{-z}\right)^{r}}{r !}(x+r)^{r} .
$$

Region of convergence The sum converges in the region $\left|\rho e^{1-\rho}\right|<1$, so considering $\rho$ as a complex variable, $\rho=x+i y$, then

$$
\left[e^{2(1-x)}\left(x^{2}+y^{2}\right)\right]^{1 / 2}<1 \text {. }
$$

From (11), the series $\sum_{n=0}^{\infty} e^{-(\tau+n)}(\tau+n)^{n} / n$ ! diverges on the boundary $\rho=1$. Consider the divergent series $\sum_{n=0}^{\infty} 1 / n$. Then by the limit comparison test

$$
\lim _{n \rightarrow \infty}\left(e^{-(c+n)} \frac{(\tau+n)^{n}}{n !} n\right)>0
$$

on utilizing Stirling's formula $n ! \sim(n / e)^{n} \sqrt{2 \pi n}$ as $n \rightarrow \infty$. The divergence of the above series can also be ascertained from the closed-form representation of a modified right-hand side in (11). 
The double pole The characteristic equation (4) may be shown to have a dominant double zero at $s=0$ for $b+c=0$ and $1+a b=0$. From the general theory of linear functional differential equations [15] it follows that there exist constants $\alpha$ and $\beta$ such that

$$
\lim _{t \rightarrow \infty}[f(t)-\alpha t]=\beta
$$

From residue theory, the constants $\alpha$ and $\beta$ can be shown to be $2 / a$ and $2 / 3$ respectively, in which case

$$
\lim _{t \rightarrow \infty}\left[f(t)-\frac{2 t}{a}\right]=\frac{2}{3}
$$

The degenerate case From (10) and (2) it can be seen that

$$
\lim _{a \rightarrow 0}\left[\sum_{n=0}^{\infty}(-1)^{n} c^{n} e^{-b(t-a n)} \frac{(t-a n)^{n}}{n !}\right]=e^{-(b+c) t}=\sum_{n=0}^{\infty} \sum_{r=0}^{n}(-1)^{n} b^{n-r} c^{n}\left(\begin{array}{l}
n \\
r
\end{array}\right) \frac{t^{n}}{n !} .
$$

This result can be ascertained directly from the differential-difference equation (1).

\section{Applications}

Two examples are investigated in which the method of the previous section is applicable.

(A) Teletraffic example Erlang in [8] considers the delay in answering telephone calls. The problem is to determine the function $f(t)$, representing the probability of the waiting time not exceeding time $t$. Hence for an $M / M / 1$ regimen Erlang shows

$$
f(t)=\int_{y=0}^{\infty} f(t+y-a) e^{-y} d y .
$$

The probability that, at the moment a call arrives, the time having elapsed since the preceding call is confined between $y$ and $y+\delta y$, is $e^{-y} d y$. The probability that the waiting time of the preceding call has been less than $(t+y-a)$ is $f(t+y-a)$, where $a$ is the connection time of a call. Differentiating the integral equation with respect to $t$ and partially integrating the result gives the differential-difference equation

$$
\left.\begin{array}{ll}
f^{\prime}(t)-f(t)+f(t-a)=0, & t \geq a \\
f^{\prime}(t)-f(t)=0, \quad f(0)=1, & 0 \leq t<a .
\end{array}\right\}
$$


The system (12) is compared with (1) where $b=-1$ and $c=1$. Hence a solution of (12) is, from Section 2,

$$
f(t)=\sum_{n=0}^{\infty}(-1)^{n} e^{t-a n} \frac{(t-a n)^{n}}{n !}=\frac{e^{\xi t}}{1-a+a \xi}
$$

in the region of convergence $\left|a e^{1-a}\right|<1$ where $\xi$ is the dominant real root of $\xi-1+e^{-a \xi}=0$. It can be shown that the characteristic equation of (12), $s-1+e^{-a s}=$ 0 has the real root distribution:

(i) One root at $s=0$ for $a \leq 0$.

(ii) One negative root plus $s=0$ for $0<a<1$.

(iii) A double (repeated) root at $s=0$ for $a=1$.

(iv) One positive root plus $s=0$ for $a>1$.

In view of the convergence criteria for the single sum $\left|a e^{1-a}\right|<1$, the following results apply for all real values of $t$

$$
\sum_{n=0}^{\infty}(-1)^{n} e^{t-a n} \frac{(t-a n)^{n}}{n !}= \begin{cases}\frac{e^{\xi t}}{1-a+a \xi} & \text { for } a>1 \\ \frac{1}{1-a} & \text { for } a<1\end{cases}
$$

Putting $t=-a \tau$, the sum can be written as

$$
\sum_{n=0}^{\infty}\left(a e^{-a}\right)^{n} \frac{(\tau+n)^{n}}{n !}= \begin{cases}\frac{e^{a \tau(1-\xi)}}{1-a+a \xi} & \text { for } a>1, \\ \frac{e^{a \tau}}{1-a} & \text { for } a<1,\end{cases}
$$

where $\xi$ is the positive root of $\xi-1+e^{-a \xi}=0$. Erlang in [8] considered only the case $0<a<1$. In the case when $a=1$ there is a double pole which, from a previous statement, results in $\lim _{t \rightarrow \infty}\{f(t)-2 t\}=2 / 3$. This fact has also been noted, in a different context, by Feller [12]. Bloom [2] proposes the problem of evaluating $\lim _{t \rightarrow \infty}\{f(t)-2 t\}$ given that, for $t$ a positive integer,

$$
f(t)=\sum_{0 \leq n \leq t} \frac{(-1)^{n} e^{t-n}(t-n)^{n}}{(n !)} .
$$

The W.M.C. problem group [27] and Holzsager [17] both solve this problem, and in particular Holzsager considers $f(t), \forall t>0$. Now, $f(t)$ satisfies the differentialdifference equation

$$
f^{\prime}(t)=f(t)-f(t-1), \quad t \geq 1 .
$$


Using the theory of linear functional differential equations, Holzsager shows that

$$
\lim _{t \rightarrow \infty}\{f(t)-2 t\}=\frac{2}{3}
$$

This work relates only to the asymptotic of the finite sum whereas in this paper it is shown that the infinite sum is equal to the asymptotic expression for all $t$.

(B) A renewal example In determining the availability of a renewed component Pages and Gondran [20] consider the case of a constant failure rate. Given that $A(t)$ is the availability of a Markovian component, $\lambda$ is the constant failure rate, and $g(t)$ is a density function, then the integro-differential equation satisfied by $A(t)$ is

$$
\frac{d}{d t} A(t)=-\lambda A(t)+\left(1-A_{0}\right) g(t)+\lambda \int_{0}^{t} g(u) A(t-u) d u, \quad A(0)=A_{0} .
$$

Taking the Laplace Transform results in

$$
\bar{A}(s)=\mathscr{L}\{A(t)\}=\frac{A_{0}+\left(1-A_{0}\right) g(s)}{s+\lambda-\lambda g(s)} .
$$

Considering the case of constant repair time, that is Mean Time To Repair, M.T.T.R., is $a$, then $g(t)=\delta(t-a)$, where $\delta(t)$ is the Impulse function. Hence,

$$
\bar{A}(s)=\frac{A_{0}+\left(1-A_{0}\right) e^{-a s}}{s+\lambda-\lambda e^{-a s}}=\sum_{n=0}^{\infty} \frac{\lambda^{n}}{(s+\lambda)^{n+1}}\left\{A_{0} e^{-a s n}+\left(1-A_{0}\right) e^{-a s(n+1)}\right\}
$$

and by inversion

$$
\begin{aligned}
A(t)= & \sum_{n=0}^{\infty} \frac{\lambda^{n}}{n !}\left\{A_{0} e^{-\lambda(t-a n)}(t-a n)^{n} H(t-a n)\right. \\
& \left.+\left(1-A_{0}\right) e^{-\lambda(t-a(n+1))}(t-a(n+1))^{n} H(t-a(n+1))\right\},
\end{aligned}
$$

where $H(x)$ is the Heaviside function. From (14) the residue at the dominant root $s=0$, of the characteristic equation $s+\lambda-\lambda e^{-a s}=0$ for $a>0$ and $1+a \lambda \neq 0$, is $1 /(1+a \lambda)$. Hence by utilizing the previous section, the result becomes

$$
\sum_{n=0}^{\infty} \frac{\lambda^{n}}{n !}\left\{A_{0} e^{-\lambda(t-a n)}(t-a n)^{n}+\left(1-A_{0}\right) e^{-\lambda(t-a(n+1))}(t-a(n+1))^{n}\right\}=\frac{1}{1+a \lambda}
$$

in its region of convergence $\left|a \lambda e^{1+a \lambda}\right|<1$ and $\forall t \in R$.

The value of the availability limit sum is independent of the initial value $A_{0}$ and the closed-form solution is independent of the value of $t$. 
It can be seen that

$$
\sum_{n=0}^{\infty} \frac{\lambda^{n}}{n !} e^{-\lambda(t-a n)}(t-a n)^{n}=\sum_{n=0}^{\infty} \frac{\lambda^{n}}{n !} e^{-\lambda(t-a(n+1))}(t-a(n+1))^{n}=\frac{1}{1+a \lambda}
$$

by putting $t-a=T$ in the second sum. Utilizing (8) and putting $t=-a \tau$ results in

$$
\sum_{n=0}^{\infty} \frac{(-1)^{n}}{n !}\left(\lambda a e^{a \lambda}\right)^{n}(\tau+n)^{n}=\frac{e^{-\lambda a \tau}}{1+a \lambda}=e^{-a \lambda \tau} \sum_{n=0}^{\infty} \sum_{r=0}^{n} \frac{(-1)^{n}}{n !}\left(\begin{array}{l}
n \\
r
\end{array}\right)(a \lambda)^{n}(\tau+r)^{n}
$$

whenever the double sum converges.

From (14) a double pole occurs at $s=0$ when $1+a \lambda=0$, and in this case

$$
\lim _{t \rightarrow \infty}\left\{A(t)+\frac{2}{a} t\right\}=\frac{2}{3}\left(3 A_{0}-2\right) .
$$

Other examples in which this technique is applicable occur for Bruwier series [1], slowing down of neutrons [23], [18] and for ruin problems in compound Poisson processes [24].

\section{Numerical examples}

The roots of the characteristic equation $s+b+c e^{-a s}=0$ can be located using Mathematica. Let $s=x+i y$ then

$$
\begin{aligned}
& R(x, y)=0=x+b+c e^{-a x} \cos a y, \\
& I(x, y)=0=y-c e^{-a x} \sin a y .
\end{aligned}
$$

Note that in (15) if for any $x, y$ is a solution then so is $-y$. Hence the non-real zeros occur in complex conjugate pairs.

Putting $t=-a \tau$, then (10) can be restated as

$$
\begin{aligned}
\sum_{n=0}^{\infty}\left(a c e^{a b}\right)^{n} \frac{(\tau+n)^{n}}{n !} & =\frac{e^{-a \tau\left(b+\xi_{1}\right)}}{1+a b+a \xi_{1}} \\
& =e^{-a b \tau} \sum_{n=0}^{\infty} \frac{(a b)^{n}}{n !} \sum_{r=0}^{n}\left(\frac{c}{b}\right)^{r}\left(\begin{array}{l}
n \\
r
\end{array}\right)(\tau+r)^{n},
\end{aligned}
$$

where $\xi_{1}$ is the dominant root of the characteristic equation.

In Table 1 , the single sum converges to the closed form term at (16) to within a truncation error of $\epsilon=10^{-12}$.

Using the technique developed by Braden [4], the single positive sum needs at least 245 terms so that its sum is within a truncation error of $\epsilon=10^{-12}$ for $(a, b, c)=$ 
TABLE 1. Examples of sums

\begin{tabular}{|c|c|r|r|}
\hline$(a, b, c)$ & \multicolumn{1}{|c|}{$\xi_{1}$} & \multicolumn{1}{c|}{ single sum at (16) } \\
\hline $0.5,0.2,0.6$ & -1.421013107061 & 2 & 8.705206947716 \\
& & -2 & 0.757217409662 \\
$8,-1,6$ & 0.997954008152 & 2 & 1.050471634335 \\
& & -2 & 0.983898536807 \\
$-4,3,1$ & -3.000006144061 & 2 & 0.999926274283 \\
& & -2 & 1.000024576849 \\
$0.1,2,-2$ & 0 & 2 & 0.558600038363 \\
& & -2 & 1.243187248034 \\
$0.15,-4,4$ & 0 & 2 & 8.300292306841 \\
& & -2 & 0.752985529781 \\
$0.99,-1,1$ & 0 & 2 & 724.274298516101 \\
& & -2 & 13.806292373109 \\
\hline
\end{tabular}

$(0.15,-4,4)$ and over one million terms for $(a, b, c)=(0.99,-1,1)$ and $\tau=2$. The double sum in (10), when it converges, generally requires many more terms in its series than does the single sum to converge to some prescribed truncation error. In Table 1 the double series converges only for the cases $(a, b, c)=(0.5,0.2,0.6)$ and $(0.1,2,-2)$.

\section{Conclusion}

A technique has been demonstrated whereby series may be represented in closed form. An association was made between a differential-difference equation and its characteristic equation, however a starting point may simply be taken as a Laplace transform equation of the type

$$
F(s)=\frac{1}{P_{n}(s)+Q_{n}(s) e^{-a s}}
$$

for $P_{n}(s)$ and $Q_{n}(s)$ polynomials in $s$. In a follow up paper, more general systems of the type

$$
\sum_{k=0}^{R}\left(\begin{array}{l}
R \\
k
\end{array}\right)(-1)^{k} f^{(k)}(t)-f(t-a)=g(t)
$$

and

$$
\sum_{k=0}^{R}\left(\begin{array}{l}
R \\
k
\end{array}\right)(-1)^{k} f^{(R-k)}(t-k a)=h(t)
$$


will be considered, together with equations that have more than one delay and equations of neutral and mixed types.

\section{Acknowledgments}

The initial inspiration leading to this work was provided to the first named author by the late Professor A. Keane. The authors also acknowledge the helpful discussions with Dr. D. Watson and Professor E. R. Love.

\section{References}

[1] R. Bellman and K. L. Cooke, Differential-Difference Equations (Academic Press, New York, 1963).

[2] D. M. Bloom, “Advanced problem 6652", American Mathematical Monthly 98 (1991) 272-273.

[3] J. M. Borwein and P. B. Borwein, "Strange series and high precision fraud", American Mathematical Monthly 99 (1992) 622-640.

[4] B. Braden, "Calculating sums of infinite series", American Mathematical Monthly 99 (1992) $649-655$.

[5] F. Brauer, “Absolute stability in delay equations", J. Diff. Eq. 69 (1987) 185-191.

[6] F. Brauer and M. Zhien, "Stability of stage-structured population models", J. Math. Anal. Appl. 126 (1987) 301-315.

[7] R. K. Brayton, "Bifurcation of periodic solutions in a nonlinear difference-differential equation of neutral type", Quart. Appl. Math. 24 (1966) 215-224.

[8] E. Brockmeyer and H. L. Halstrom, The Life and Works of A. K. Erlang (Copenhagen, 1948).

[9] K. L. Cooke and Z. Grossman, "Discrete delay, distributed delay and stability switches", J. Math. Anal. Appl. 86 (1982) 592-627.

[10] K. L. Cooke and P. van den Driessche, "On zeros of some transcendental equations", Funkcialaj Ekvacioj 29 (1986) 77-90.

[11] R. D. Driver, D. W. Sasser and M. L. Slater, "The equation with "small" delay", American Mathematical Society 80 (1973) 990-995.

[12] W. Feller, An Introduction to Probability Theory and its Applications (John Wiley and Sons, Chichester, 1971).

[13] M. K. Grammatikopoulos and I. P. Stravroulakis, "Necessary and sufficient conditions for oscillation of neutral equations with deviating arguments", J. London Math. Soc. (2) 41 (1990) 244-260.

[14] J. K. Hale, Homoclinic Orbits and Chaos in Delay Equations, Pitman Research Lecture Notes 157 (Pitman, 1997).

[15] J. K. Hale and S. M. Yerduyn Lunel, Introduction to Functional Differential Equations (Springer, New York, 1993).

[16] D. Y. Hao and F. Brauer, "Analysis of a characteristic equation", J. Integral Eq. Appl. 3 (1991) 239-253.

[17] R. Holzsager, "Solution to asymptotic linearity", American Mathematical Monthly 99 (1992) 878-880.

[18] A. Keane, "Slowing down from an energy distributed neutron source", Nuclear Sci. Eng. 10 (1961) 117-119. 
[19] N. Minorsky, Nonlinear Oscillations (D. Van Nostrand Company, Princeton, 1962).

[20] A. Pages and M. Gondran, System Reliability Evaluation and Prediction in Engineering (North Oxford Academic, 1986).

[21] L. S. Pontryagin, "On the zeros of some elementary transcendental functions", American Math. Soc. Trans., Ser. 21 (1955) 95-110.

[22] G. Seifert, On an Interval Map Associated with a Delay Logistic Equation with Discontinuous Delays, Lecture Notes in Mathematics, 1475 (Springer, Berlin, 1991).

[23] T. Teichmann, “Slowing down of neutrons”, Nuclear Sci. Eng. 7 (1960) 291-294.

[24] H. C. Tijms, Stochastic Modelling and Analysis : A Computational Approach (John Wiley and Sons, Chichester, 1986).

[25] F. S. Wheeler, "Two differential-difference equations arising in number theory", Trans. American Math. Soc. 318 (1990) 491-523.

[26] E. T. Whittaker and G. N. Watson, A Course of Modern Analysis, Fourth Edition, reprinted (Cambridge University Press, Cambridge, 1978).

[27] W. M. C. problem group, "Solution to asymptotic linearity", American Mathematical Monthly 99 (1992) 877-878.

[28] E. M. Wright, "Stability criteria and the real roots of a transcendental equation", J. Soc. Indust. Appl. Math. 9 (1961) 136-148. 